\title{
Advantages of the net benefit regression framework for trial-based economic evaluations of cancer treatments: an example from the Canadian Cancer Trials Group CO.17 trial
}

\author{
Jeffrey S. Hoch* (1), Annette Hay, Wanrudee Isaranuwatchai, Kednapa Thavorn, Natasha B. Leighl, Dongsheng Tu, \\ Logan Trenaman, Carolyn S. Dewa, Chris O'Callaghan, Joseph Pater, Derek Jonker, Bingshu E. Chen and \\ Nicole Mittmann
}

\begin{abstract}
Background: Economic evaluations commonly accompany trials of new treatments or interventions; however, regression methods and their corresponding advantages for the analysis of cost-effectiveness data are not widely appreciated.
\end{abstract}

Methods: To illustrate regression-based economic evaluation, we review a cost-effectiveness analysis conducted by the Canadian Cancer Trials Group's Committee on Economic Analysis and implement net benefit regression.

Results: Net benefit regression offers a simple option for cost-effectiveness analyses of person-level data. By placing economic evaluation in a regression framework, regression-based techniques can facilitate the analysis and provide simple solutions to commonly encountered challenges (e.g., the need to adjust for potential confounders, identify key patient subgroups, and/or summarize "challenging" findings, like when a more effective regimen has the potential to be cost-saving).

Conclusions: Economic evaluations of patient-level data (e.g., from a clinical trial) can use net benefit regression to facilitate analysis and enhance results.

Keywords: Net benefit regression, Economic evaluation, Cost-effectiveness

\section{Background}

We must deal with the escalating price of cancer therapy now... We cannot ignore the cumulative costs of the tests and treatments we recommend and prescribe. As the agents of change, professional societies, including their academic and practicing oncologist members, must lead the way. The time to start is now [1].

Cancer is a costly disease; there are huge costs physically, mentally and financially. A major component of many treatment regimens is pharmaceuticals. Fiscal

\footnotetext{
* Correspondence: jshoch@ucdavis.edu

Division of Health Policy and Management, Department of Public Health Sciences and Associate Director, Center for Healthcare Policy and Research, 2103 Stockton Blvd, Sacramento, CA 95817, USA
}

toxicity of cancer treatment is not unique to patients and their families; healthcare payers also experience financial distress. Without the resources to pay for all treatments for all diseases for all patients, most healthcare payers have embraced an evidence informed decision-making process involving recommendation committees. Frequently, these recommendation committees embrace other types of evidence in addition to clinical evidence. For example, in Canada, the panCanadian Oncology Drug Review (pCODR), a national recommendation committee for oncology drugs, uses a deliberative framework that includes clinical evidence, patient values, system feasibility as well as economic evidence [2]. In the United States, the Institute for

(c) The Author(s). 2019 Open Access This article is distributed under the terms of the Creative Commons Attribution 4.0 International License (http://creativecommons.org/licenses/by/4.0/), which permits unrestricted use, distribution, and 
Clinical and Economic Review considers both net clinical benefit as well as value (i.e., cost-effectiveness and budget impact). Usually, the economic evidence used by recommendation committees is in the form of a costeffectiveness model with inputs, based in part, on patient-level trial data.

In advance of formal drug reimbursement dossier submissions trial data are often presented at national conferences and published in scientific journals, providing an initial (and often impactful) preview of the clinical and economic evidence. Thus, cost-effectiveness analyses based entirely on patient-level trial data have the potential to play a major role in influencing clinical and decision maker perceptions of whether a drug provides value (e.g., is economically attractive). The analysis of a costeffectiveness dataset provides insight into the value of the clinical benefit, over the same time horizon as the clinical study. In this way, the extra costs of the extra patient benefits accruing in the trial can be appreciated concurrently. However, there are some challenges that attend the analysis of patient-level cost-effectiveness data. For example, in cancer studies, these can involve the need to i) adjust for potential confounders, ii) identify key patient subgroups (e.g., with biomarkers), and iii) summarize the economic evidence when there is a negative cost-effectiveness ratio (e.g., when more effective treatment regimens are also potentially cost saving).

This article illustrates a regression-based method for analyzing patient-level cost-effectiveness data called net benefit regression. It has a variety of benefits that address shortcomings in conventional cost-effectiveness analysis methods. These benefits are illustrated using the Canadian Cancer Trials Group CO.17 study showing that patients with advanced colorectal cancer had improved overall survival and greater costs when cetuximab, an epidermal growth factor receptor-targeting antibody, was given in addition to best supportive care. Although the concepts of net benefit and net benefit regression have been applied in other healthcare areas, their application in oncology has not been widespread [3-5]. It is the goal of this article to clarify how to use and interpret the net benefit regression method, so that more authors and readers can appreciate what it offers.

\section{Methods}

\section{Case study description}

Mittmann and colleagues [6] conducted an economic evaluation of cetuximab plus best supportive care versus best supportive care alone in unselected advanced colorectal cancer patients. The initial clinical trial was conducted by the Canadian Cancer Trials Group as a multicenter, open-label, randomized phase III trial of cetuximab plus best supportive care versus best supportive care alone in patients with chemotherapy-refractory metastatic EGFR-positive colorectal cancer (ClinicalTrials.gov number NCT00079066). Survival times for the entire study population and for patients whose tumors harbored wild-type $K R A S$ were calculated over an 18- to 19-month period [6], and the trial (hereafter referred to as CO.17) found a statistically significant overall survival advantage for cetuximab with a 1.5 month difference in median survival for cetuximab versus best supportive care [7]. In patients with wild-type KRAS tumors, there was a larger survival advantage (i.e., 4.7 months additional median survival for cetuximab) [8].

Mittmann and colleagues conducted a cost-effectiveness analysis using prospectively collected cost and quality adjusted life year (QALY) data for patients in the CO.17 [6]. For patients in the trial, cetuximab showed unattractively high incremental cost-effectiveness ratios. The incremental cost-effectiveness ratios (ICERs) were more favorable for patients whose tumors harbored wild-type KRAS but were still more than $\$ 186,000$ per quality-adjusted lifeyear gained. Since there is no universally agreed upon cost-effectiveness threshold or willingness to pay (WTP) value, jurisdictions often adopt fuzzy thresholds that are guided by several factors [9-11]. Nevertheless, the likelihood of a positive funding recommendation appears inversely related to the incremental cost-effectiveness ratio (i.e., higher ICERs have a lower probability of being funded). [12, 13] This suggest that cost-effectiveness methods that explicitly allow the WTP threshold to vary may be helpful.

In the following section, we describe net benefit regression before applying the technique to analyze the cost-effectiveness data for patients in the CO.17 study.

\section{Net benefit regression framework}

We briefly review below the key components of net benefit regression and offer additional references for the interested reader [14-16]. With the net benefit regression approach, analysts can use regression-based techniques to analyze cost-effectiveness data; some advantages of the net benefit regression approach include facilitating solutions to challenging statistical situations (e.g., negative cost-effectiveness ratios or when Fieller's theorem will not yield a confidence interval) [14]. The net benefit regression framework was proposed a decade ago to marry regression and cost-effectiveness methods [17]. At that time, the conventional statistic reported in most cost-effectiveness studies was the ICER.

\section{Building from the ICER}

Mathematically, the ICER estimate is defined as Extra Cost $\div$ Extra Effect, where Extra Cost is defined as $\Delta \mathrm{C}=$ Expected Cost with New Treatment - Expected Cost with Usual Care and Extra Effect is defined as $\Delta \mathrm{E}=$ Expected Effect with New Treatment - Expected Effect with Usual 
Care. With a cost-effectiveness dataset, it is common to use the Average Cost and Average Effect to represent Expected values. The ICER is troublesome to estimate because it is a ratio; however, its parts-the numerator and denominator-can be estimated easily by regression.

If one defines a binary treatment indicator variable as $\mathrm{TX}=1$ for a study participant receiving the new treatment, and $\mathrm{TX}=0$ for a study participant receiving usual care, then one can use ordinary least squares (OLS) to estimate linear regressions for cost $\left(\mathrm{c}_{\mathrm{i}}\right)$ and effect $\left(\mathrm{e}_{\mathrm{i}}\right)$. By adding an interaction term (say, between the KRAS status and TX indicator variables), it is possible to explore hypothesis-generating questions about subgroups for whom the new intervention may be more (or less) cost-effective. For example, is a drug more cost-effective for patients with wild-type KRAS tumors?

\section{Willingness to pay (WTP)}

When a new treatment costs more $(\Delta C>0)$ and is more effective $(\Delta \mathrm{E}>0)$, the ICER $>0$. For decisions, an ICER must be compared with a WTP threshold value. Unfortunately, a decision maker's WTP is unknown, so methods that treat WTP as unknown are best (e.g., varying WTP and exploring how a recommendation based on the estimated ICER may change). Net benefit regression addresses the unknown nature of the "correct" WTP value within the incremental net benefit.

\section{Incremental net benefit regression}

By computing each patient's net benefit (NB) as WTP $\times$ $\mathrm{e}_{\mathrm{i}}-\mathrm{c}_{\mathrm{i}}$ and using it for a dependent variable, one can run a simple or multiple linear regression of the form

$$
\begin{aligned}
\mathrm{NB}= & \mathrm{b}_{0}+\mathrm{b}_{\mathrm{TX}} \mathrm{TX}+\varepsilon_{\mathrm{NB}} \\
\mathrm{NB}= & \mathrm{b}_{0}+\mathrm{b}_{\mathrm{TX}} \mathrm{TX}+\mathrm{b}_{1} \mathrm{X}_{1}+\cdots \\
& +\mathrm{b}_{\mathrm{p}} \mathrm{X}_{\mathrm{p}}+\varepsilon_{\mathrm{NB}},
\end{aligned}
$$

(respectively)

If $b_{T X}>0$, the new treatment is cost-effective since $b_{T X}$ equals the incremental net benefit (INB); the INB conveys by how much the value of the extra effect outweighs the extra cost (i.e., INB $=\mathrm{WTP} \times \Delta \mathrm{E}-\Delta \mathrm{C}$ ) [17]. Another way to view the INB is as the difference in the average net benefits between the new treatment and usual care: new treatment is more cost-effective if it has higher net benefits than usual care. The linearity of the dependent variable NB means the estimate of $b_{T X}=$ WTP $\times \Delta \mathrm{E}-\Delta \mathrm{C}$. While the $95 \%$ confidence interval (CI) for the ICER cannot be made from the separate CIs for the estimates of $\Delta \mathrm{C}$ and $\Delta \mathrm{E}$ (because this process ignores the correlation between the cost and effect data) [18], the $95 \%$ CI for $b_{\mathrm{TX}}$ is the $95 \%$ CI for the INB. If there is concern about using a parametric method for the $95 \% \mathrm{CI}$, one can use a non-parametric method like bootstrapping [19].
By estimating net benefit regression equations with various WTP values, one can gauge the sensitivity of cost-effectiveness findings in relation to WTP assumptions. One WTP value that should always be checked in a net benefit regression is WTP $=\$ \Delta \mathrm{C} / \Delta \mathrm{E}$ since this should yield an INB estimate of zero (i.e., $b_{T X}=0$ ). By setting WTP $=\$ 0$, the INB should become $-1 \times \Delta$ C. One can characterize uncertainty using CIs or $p$-values to create cost-effectiveness acceptability curves (e.g., see [20] for a step by step tutorial on using $p$-values this way). Because the INB and the ICER are related through WTP, both their estimates and uncertainty are closely connected. A graph of INB by WTP has a y-intercept equal to $-\Delta C$, a slope of $\Delta E$ and an $x$-intercept of the ICER. The addition to the graph of $95 \%$ CIs for the INB illustrates, at their $\mathrm{x}$-intercepts, the lower and upper 95\% CIs (from Fieller's Theorem) for the ICER (see Results section for examples). We illustrate these points next using net benefit regression results.

\section{Results}

Table 1 reports the results of simple linear regressions with dependent variables Effect, Cost and NB regressed on the cetuximab treatment indicator (i.e., the TX variable in the METHODS section). The estimates in Table 1 represent $\Delta \mathrm{E}, \Delta \mathrm{C}$ and $\triangle \mathrm{NB}$ (i.e., INB), respectively. Results when WTP $=\$ 0$ are reported in the NB ( $\$ 0)$ column; results for WTP $=\$ 500,000$ are reported in the NB $(\$ 500 \mathrm{k})$ column. In this economic analysis, cetuximab showed extra cost of $\$ 22,210$ and extra effect of 0.0771 QALYs (see the row labeled "ALL" in Table 1) when compared with best supportive care for all patients in the CO.17 trial. This corresponds to an ICER over $\$ 288,000$ (i.e., 22,210/0.0771), not generally considered economically attractive. However, the results differ by KRAS status. While the extra cost estimate appears larger for patients with wild-type $K R A S$ tumors $(\Delta C=\$ 30,843, p$-value $<0.001)$ than patients whose tumors do not express wild-type $K R A S$ $(\Delta \mathrm{C}=\$ 13,787, p$-value $<0.001)$, the extra effect estimates tell a much different story. Cetuximab appears more effective than best supportive care for patients with wild-type KRAS tumors $(\Delta \mathrm{E}=0.1769$ QALYs, $p$-value $<0.001)$ but less effective than best supportive care for patients whose tumors do not express wild-type KRAS $(\Delta \mathrm{E}=-0.0172$ QALYs, $p$-value $>0.40$ ).

Table 2 shows the estimates for multiple linear regression; these results further support analyzing the data stratified by KRAS status. The coefficient on the cetuximab treatment indicator, which represents INB for patients with mutant status, is negative for WTP values from $\$ 0$ to $\$ 500,000$. However, the interaction term between cetuximab treatment and wild-type KRAS status, which represents difference of INBs between patients with KRAS wild-type and mutant statuses, switches from 


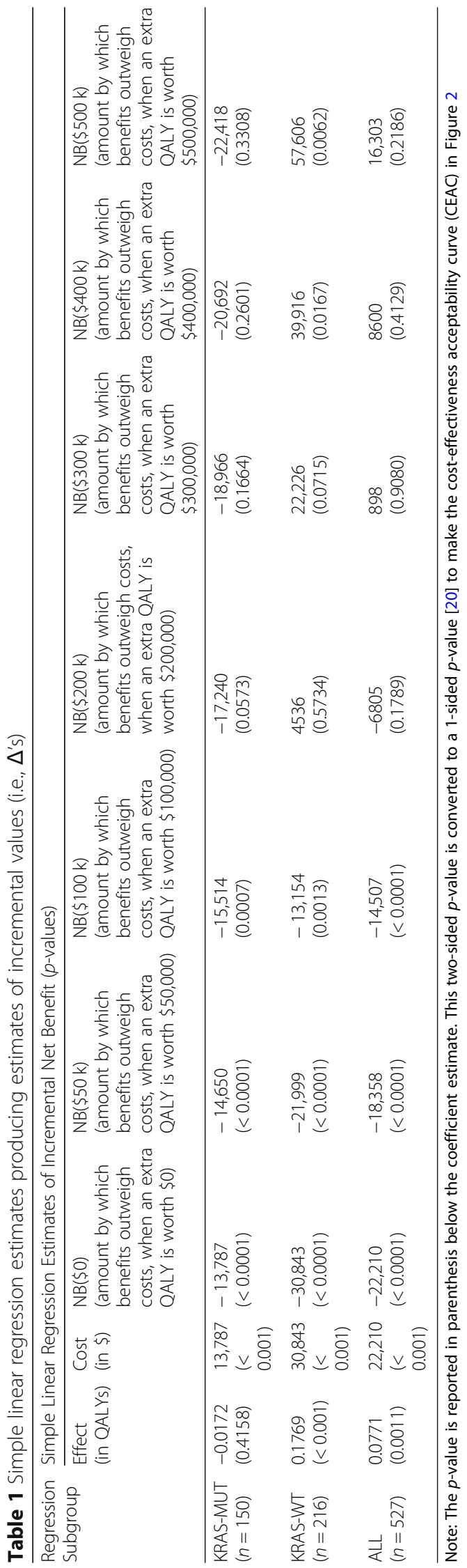




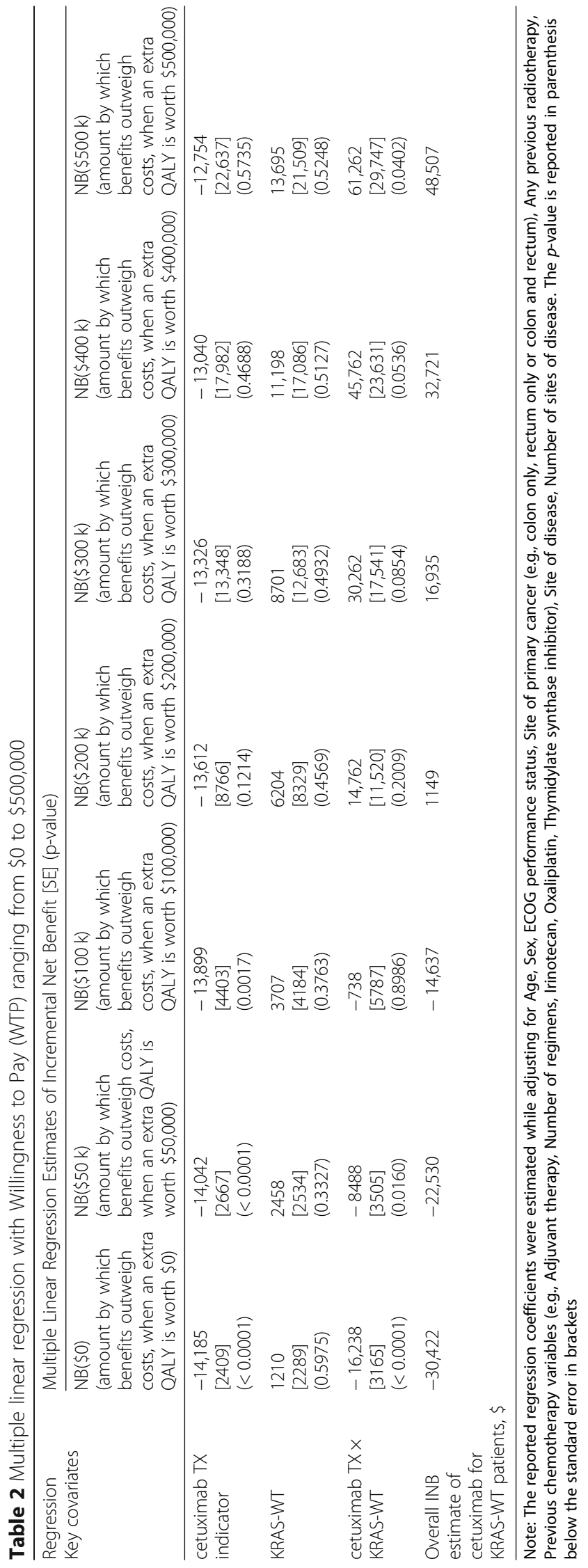




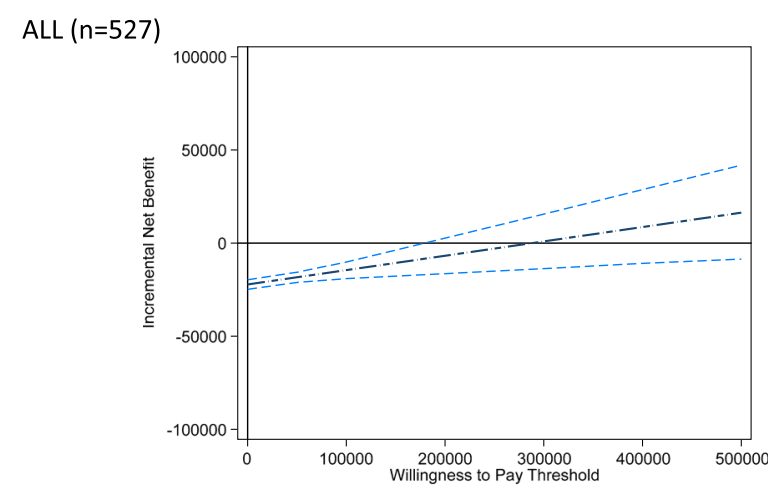

KRAS-Wild Type $(\mathrm{n}=216)$

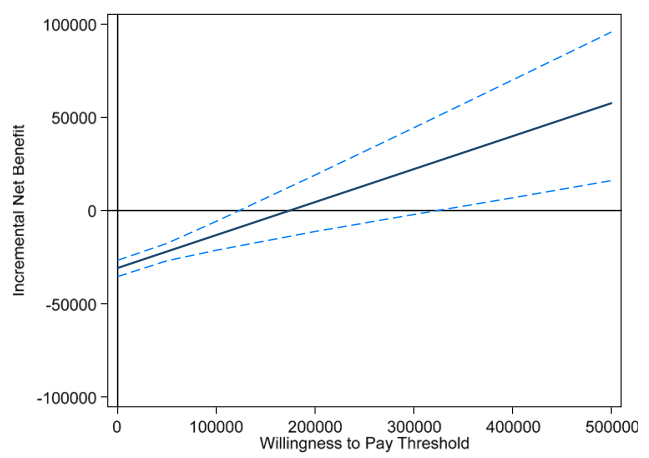

KRAS-Mutant $(\mathrm{n}=150)$

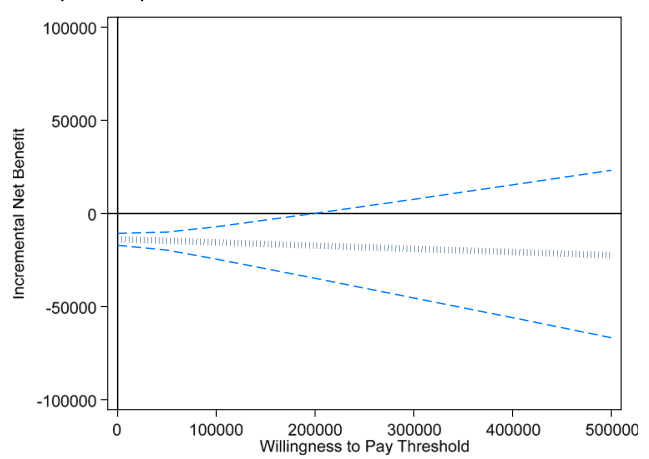

Fig. 1 Incremental net benefit estimate for all patients (upper graph, dashed line), KRAS-WT (middle graph, solid line), and KRAS-MUT (lower graph, hashed line) and 95\% confidence intervals (dashed lines)

a negative value $(-738)$ to a positive value $(14,762)$ as WTP increases from $\$ 100,000$ to $\$ 200,000$. This coincides with the INB estimate transitioning from a negative value $(-14,637)$ to a positive value $(1149)$ over the same WTP range for patients whose tumors express wild-type KRAS (KRAS-WT). For the lowest value of WTP (\$0), the interaction term between the cetuximab treatment and KRAS-WT indicator variables is statistically significantly negative $(-16,238, p$-value $<0.001)$. This implies that $\triangle \mathrm{C}$ is significantly higher for patients with wild-type KRAS than those with mutant KRAS. Conversely, for the highest value of WTP $(\$ 500,000)$, the interaction term is statistically significantly positive (61, $262, p$-value $<0.05$ ); this suggests INB with this WTP is significantly higher for patients with wild-type KRAS than those with mutant KRAS.

Figure 1 plots the incremental net benefit estimate (as a solid line) and the pointwise $95 \%$ CIs (as dashed lines) in relation to WTP values varying from $\$ 0$ to $\$ 500,000$. Vertical values greater than zero indicate when INB is positive and cetuximab is cost-effective. The graphs for the overall sample and the KRAS-WT sub-group (the top and middle graphs in Fig. 1) show a positively sloped INB line that intersects the horizontal axis; for the overall sample this occurs near the WTP value of $\$ 300,000$ and near $\$ 200,000$ for the KRAS-WT subgroup. For the patients whose tumors do not express wild-type KRAS (i.e., the KRAS-MUT group), the negatively sloped INB line does not intersect any positive WTP value (on the horizontal axis). Figure 2 communicates the probability that cetuximab is cost-effective as WTP varies. There are three curves: one for KRAS-WT patients (upper solid line), one for all patients (middle dashed line) and one for KRAS-MUT patients (lower hashed line).

\section{Discussion}

Typically, ICERs are the metrics reported in economic evaluations; However, in this case study, the ICER for the patients whose tumors do not express wild-type KRAS (KRAS-MUT) is negative. Based on expert recommendations, this means the ICER should not be calculated [21]. This makes it challenging to report the conventional cost-effectiveness statistic (which is negative in this case) and to report its 95\% CI (where at least one limit will be negative as well). In contrast, Table 1's negative INB estimates, reported for all WTP values, indicate that cetuximab for KRAS-MUT patients is not economically attractive (at least for WTP values from $\$ 0$ to $\$ 500,000)$. For KRAS-WT patients, the INB estimate becomes positive (switching from $-13,154$ to 4536 ) as WTP increases from $\$ 100,000$ to $\$ 200,000$. This indicates that the ICER falls within this range $(\triangle \mathrm{C} / \Delta \mathrm{E}=30$, $843 / 0.1769 \approx 174,350$ per QALY). The overall sample demonstrates a similar pattern, switching from -6805 to 898 as the WTP increases from $\$ 200,000$ to $\$ 300,000$ due to the overall ICER being $\Delta \mathrm{C} / \Delta \mathrm{E}=22,210$ / $0.0771 \approx \$ 288,000$ per QALY. As noted earlier, when $\mathrm{WTP}=\$ 0$, the INB estimate reduces to $-\Delta C$; this explains the similarity between the coefficients in the Cost Column and those in the $\mathrm{NB}(\$ 0)$ column in Table 1.

As noted earlier, the findings in Table 2 support stratifying the analysis by KRAS status. Either simple linear or multiple linear regression can be run separately stratifying on a patient's tumor's KRAS status. In this case study, we simplified matters by focusing on simple linear regressions (except for Table 2). The findings of the 


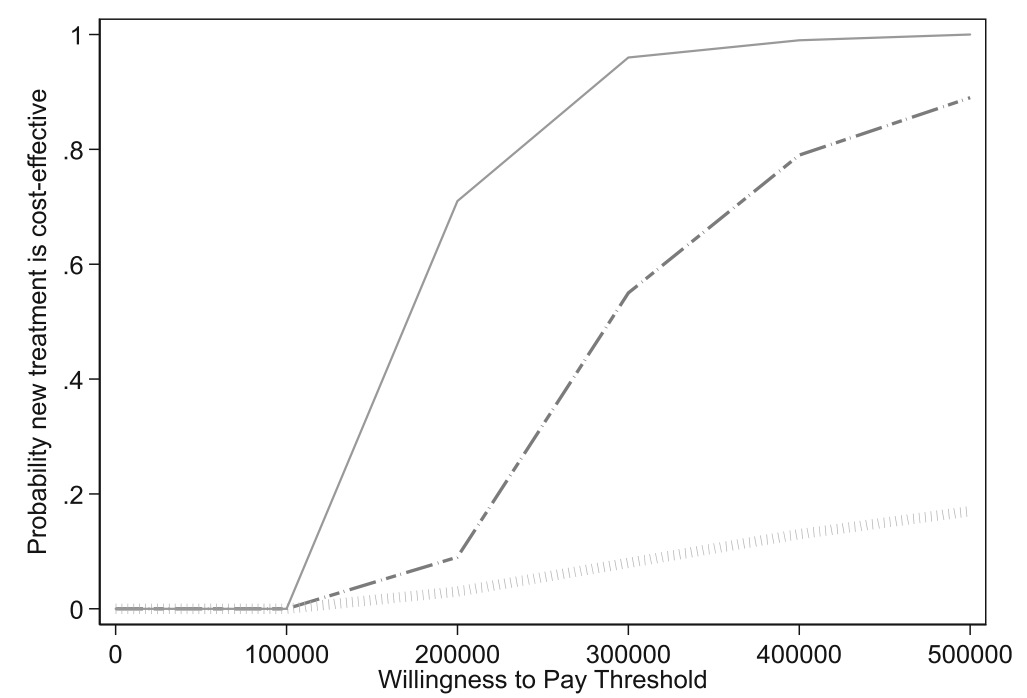

Fig. 2 Probability that new treatment is cost-effective for KRAS-WT (upper solid line), all patients (middle dashed line) and KRAS-MUT (lower hashed line) by Willingness to Pay threshold values

simple linear regression models were similar to those of the multiple linear regression models for small WTP values; they diverged more for larger WTP values. This suggests that there is important variability in the patient outcome related to the independent variables; however, the variability in cost is not as strongly associated with the independent variables since adjusting for the patient covariates (i.e., all of the $\mathrm{X}_{\mathrm{p}}{ }^{\prime} \mathrm{s}$ ) does not affect the INB estimate for small WTP values. In passing, we note that investigators interested in studying a patient subgroup, defined by a continuous variable (e.g., age, disease severity, etc.), would not be able to stratify and run separate models; a model with a treatment interaction term would be better suited to exploring this type of hypothesis generating question (involving a continuous covariate).

Figure 1 demonstrates the usefulness of an INB by WTP graph. The different shapes of the curves suggest different findings. The upper graph (for the overall sample) and the middle graph (for the KRAS-WT group) show INB lines with negative $y$-intercepts, positive slopes and $\mathrm{x}$-intercepts at WTP values of approximately $\$ 300,000$ (for the overall sample) and approximately $\$ 200,000$ (for the KRAS-WT group). As noted in the Methods section, an INB by WTP graph has a yintercept equal to $-\Delta \mathrm{C}$, a slope of $\Delta \mathrm{E}$ and an $\mathrm{x}$-intercept of the ICER. Thus, a negative y-intercept means cetuximab is more costly, a positive slope means that cetuximab is more effective, and the WTP value where the INB estimate line intersects is the ICER. Of the three graphs in Fig. 1, the KRAS-WT group has the steepest INB estimate line; therefore, that group enjoys the largest gain from treatment (i.e., has the biggest $\Delta \mathrm{E}$ ). For the KRAS-MUT group, the negative y-intercept means cetuximab is more costly, the slightly negative slope means that cetuximab is slightly less effective than best supportive care, and the WTP value where the INB estimate line looks to intersect indicates a negative ICER.

Figure 1 can also be used to characterize the uncertainty associated with both the INB and the ICER. For the KRAS-WT group (in the middle graph), the upper and lower 95\% confidence limits for the INB intersect the horizontal axis over the illustrated WTP range of $\$ 0$ to $\$ 500,000$. The two intersection points mark the Fieller's Theorem 95\% CI for the ICER. This 95\% CI corresponds very closely to the $95 \%$ CI of $\$ 130,326$ to 334 , 940 reported in the original economic analysis. For the overall sample, Mittmann and colleagues reported a 95\% CI of $\$ 187,440$ to 898,201 . This is congruent with the upper graph in Fig. 1; one confidence limit intersects the horizontal axis near a WTP $=\$ 200,000$ and the other intersection point appears greater than $\$ 500,000$. The lower graph (for the KRAS-MUT group) suggests a negative ICER with one $95 \%$ confidence limit that will be negative.

The cost-effectiveness acceptability curve (CEAC) in Fig. 2 combines parts of the regression results and Fig. 1 to characterize uncertainty [20]. The CEAC shows the probability that cetuximab plus best supportive care is cost-effective compared to best supportive care alone. WTP varies along the horizontal axis reflecting its unknown nature (to the analyst). The vertical axis communicates the portion of the INB distribution that is positive (indicating the probability that cetuximab is cost-effective). The three curves-one for KRAS-WT patients (upper solid line), one for all patients (middle dashed line) and one for KRAS-MUT patients (lower 
solid line)-support the general conclusions that have been offered. While the CEACs presented in Fig. 2 were made using parametric $p$-values from Table 1 , it is possible to create them using non-parametric bootstrapping methods [20].

\section{Limitations}

We conclude our discussion by reviewing some key limitations in our example involving the analysis of person-level cost-effectiveness data. The usefulness of person-level costeffectiveness data is diminished when either a relevant outcome is not included in the original study or when the trial is too short in duration to see activity in the outcome of interest. The original clinical trial in our example used overall survival as its primary end point with secondary outcomes that included progression-free survival as well as quality adjusted life years (QALYs). Even with the strength of the trial's design, there is still the critical question of whether "enough" study participants contributed outcome data. Of the randomly assigned 572 patients, a total of 456 deaths occurred by the date of analysis. The median survival was 6.1 months in the cetuximab group and 4.6 months in the supportive-care group. The proportions of patients surviving at 6 and 12 months were 50 and 21\%, respectively, in the cetuximab group and 33 and 16\%, respectively, in the supportive care group.

Typically, when time-to-event data (e.g., survival) are incomplete, methods for censored data are employed. In contrast to the original economic evaluation which employed two methods to calculate overall survival: the restricted mean survival method (which restricts calculation of mean survival to the longest observed survival time) and the Kaplan - Meier method (which takes into account censoring), we used only the restricted mean survival method. Our simplifications (e.g., ordinary least squares to estimate a simple linear regression without specific methods for censoring) did not appear to make any qualitative difference in this case study; the original economic evaluation reported ICERs of $\$ 186,761$ (KRAS-WT) and $\$ 299,613$ (entire study population) compared to ICERs of $\$ 174,353$ and $\$ 288,067$ calculated using estimates from our Table 1, respectively. While our simple illustration of net benefit regression is meant to facilitate understanding, there are situations where more advanced methods for the analysis of censored cost-effectiveness data may be desired. Advanced papers by Bang and Tsiatis [22] as well as Chen et al. [23] provide excellent direction in this area. More advanced methods for simultaneous estimation of cost and effect equations are also available [24].

Finally, often analysts do not report INB results for all willingness to pay (WTP) values. When a reader is interested in a WTP value occurring within the range of WTP values that are used (e.g., WTP $=\$ 123,456$ ) or a
WTP value outside the range (e.g. WTP $=\$ 600,000$ ), this may appear to be a concern. This concern can be addressed easily because the formula for INB is linear (i.e., $\mathrm{INB}=\mathrm{WTP} \times \Delta \mathrm{E}-\Delta \mathrm{C}$ ). For each $\$ 1$ change in WTP, the INB changes by $\Delta \mathrm{E}$. Thus, the INB when WTP $=\$ 123$, 456 is $\$ 23,456 \times \Delta \mathrm{E}$ more than the INB when WTP = $\$ 100,000$. Using Table 1 and KRAS-WT as an example, $\operatorname{INB}(\$ 123,456)=\operatorname{INB}(\$ 100,000)+(\$ 23,456 \times \Delta \mathrm{E})=-13$, $154+(\$ 23,456 \times 0.1769)=-\$ 9005$. This matches the result from a direct calculation of $\operatorname{INB}(\$ 123,456)=\$ 123$, $456 \times \Delta \mathrm{E}-\Delta \mathrm{C}=\$ 123,456 \times 0.1769-\$ 30,843 \approx-\$ 9005$.

This method can also be used for WTP values outside of the WTP ranges reported. For example, using the values in Table 1 for KRAS-WT, $\operatorname{INB}(\$ 600,000)=\operatorname{INB}(\$ 500,000)$ $+(\$ 100,000 \times \Delta \mathrm{E})=57,606+(\$ 100,000 \times 0.1769)=\$ 75,296$.

Direct calculation verifies this result for KRAS-WT, $\operatorname{INB}(\$ 600,000)=\$ 600,000 \times 0.1769-\$ 30,843 \approx \$ 75,297$.

\section{Conclusion}

This article showcases the advantages of the net benefit regression framework [17]. The framework allows incremental cost and incremental effect to be estimated either separately (i.e., using cost or effect as a dependent variable) or together (i.e., using net benefit as a dependent variable). In this paper's case study, there was a straightforward application of OLS. However, more ambitious analytical strategies with more sophisticated techniques can be used (e.g., using regression diagnostics, employing interaction terms and/or using advanced methods for non-randomized data). We were able to adjust our cost-effectiveness analysis for covariates using multiple linear regression and to explore clinically relevant patient subgroups. The incremental net benefit by WTP curve illustrated both our estimate of cost-effectiveness and the associated uncertainty. The INB by WTP graph allows the cost-effectiveness results to reflect the unknown WTP's impact on policy implications. When analyzing a cost-effectiveness dataset, net benefit regression can be a useful starting point for exploring one's data and communicating a new treatment's value.

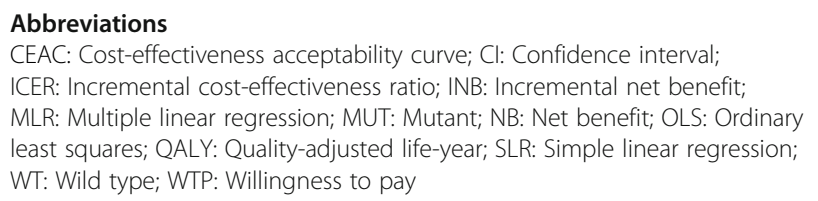

Acknowledgements

Not applicable.

Authors' contributions

AH, NBL, DT, CO'C, JP, DJ, BEC, NM designed and conducted the randomized trial that served as the case study for this analysis. JSH, KT and Wl led the net benefit regression analysis. JSH, AH, LT, CD and WI collaborated on drafting the manuscript. All authors provided feedback on the manuscript. All authors approved the manuscript for submission. 


\section{Funding}

This work was supported by funding from the Canadian Centre for Applied Research in Cancer Control (ARCC). ARCC is funded by a grant from the Canadian Cancer Society Research Institute (CCSRI). The Canadian Cancer Trials Group is supported in part by the CCSRI. Funding for this trial used in the examples was provided by Eli Lilly Canada, Hoffman-La Roche Limited, Bristol-Myers Squibb, and ImClone Systems Incorporated. The authors take responsibility for all aspects of the study including design, data acquisition, analysis, interpretation, and drafting of the article. The study funders played no role in the design, analysis, or interpretation of the study.

\section{Availability of data and materials}

The datasets analysed during the current study are not publicly available due to the Canadian Cancer Trials Group's policy. However, the results can verified based on this analysis and the separate publication of the original cost-effectiveness article.

\section{Ethics approval and consent to participate}

This was a reanalysis of the data from a published paper, so ethics approval was not sought by the research ethics boards of participating institutions.

\section{Consent for publication}

Not applicable.

\section{Competing interests}

The authors declare that they have no competing interests. The authors take responsibility for all aspects of the study including design, data acquisition, analysis, interpretation, and drafting of the article.

Received: 11 December 2018 Accepted: 31 May 2019

Published online: 07 June 2019

\section{References}

1. Fojo T, Grady C. How much is life worth: cetuximab, non-small cell lung cancer, and the $\$ 440$ billion question. J Natl Cancer Inst. 2009;101(15):1044-8.

2. Hoch JS, Sabharwal M. Informing Canada's cancer drug funding decisions with scientific evidence and patient perspectives: the Pan-Canadian oncology drug review. Curr Oncol. 2013;20(2):121-4.

3. Thavorn K, Coyle D, Hoch JS, Vandermeer L, Mazzarello S, Wang Z, Dranitsaris G, Fergusson D. Clemons M. a cost-utility analysis of risk modelguided versus physician's choice antiemetic prophylaxis in patients receiving chemotherapy for early-stage breast cancer: a net benefit regression approach. Support Care Cancer. 2017.

4. Lairson DR, Dicarlo M, Deshmuk AA, Fagan HB, Sifri R, Katurakes N, Cocroft J, Sendecki J, Swan H, Vernon SW, Myers RE. Cost-effectiveness of a standard intervention versus a navigated intervention on colorectal cancer screening use in primary care. Cancer. 2014;120(7):1042-9.

5. Shih YC, Pan IW, Tsai YW. Information technology facilitates costeffectiveness analysis in developing countries: an observational study of breast cancer chemotherapy in Taiwan. Pharmacoeconomics. 2009;27(11): 947-61.

6. Mittmann N, Au HJ, Tu D, O'Callaghan CJ, Isogai PK, Karapetis CS, Zalcberg JR, Evans WK, Moore MJ, Siddiqui J, Findlay B, Colwell B, Simes J, Gibbs P, Links $\mathrm{M}$, Tebbutt NC, Jonker DJ. Working group on economic analysis of National Cancer Institute of Canada clinical trials group.; Australasian gastrointestinal interest group. Prospective cost-effectiveness analysis of cetuximab in metastatic colorectal cancer: evaluation of National Cancer Institute of Canada clinical trials group CO.17 trial. J Natl Cancer Inst. 2009; 101(17):1182-92

7. Jonker DJ, O'Callaghan CJ, Karapetis CS, Zalcberg JR, Tu D, Au HJ, Berry SR, Krahn M, Price T, Simes RJ, Tebbutt NC, van Hazel G, Wierzbicki R, Langer C, Moore MJ. Cetuximab for the treatment of colorectal cancer. N Engl J Med. 2007 Nov 15;357(20):2040-8.

8. Karapetis CS, Khambata-Ford S, Jonker DJ, O'Callaghan CJ, Tu D, Tebbutt NC, Simes RJ, Chalchal H, Shapiro JD, Robitaille S, Price TJ, Shepherd L, Au HJ, Langer C, Moore MJ, Zalcberg JR. K-ras mutations and benefit from cetuximab in advanced colorectal cancer. N Engl J Med. 2008:359(17):1757-65.

9. Rocchi A, Menon D, Verma S, Miller E. The role of economic evidence in Canadian oncology reimbursement decision-making: to lambda and beyond. Value Health. 2008;11(4):771-83.
10. Eichler HG, Kong SX, Gerth WC, Mavros P, Jonsson B. Use of costeffectiveness analysis in health-care resource allocation decision-making: how are cost-effectiveness thresholds expected to emerge? Value Health. 2004:7(5):518-28.

11. Rawlins MD, Culyer AJ. National Institute for clinical excellence and its value judgments. BMJ. 2004;329(7459):224-7.

12. Dakin H, Devlin N, Feng Y, Rice N, O'Neill P, Parkin D. The influence of costeffectiveness and other factors on nice decisions. Health Econ. 2015;24: 1256-71.

13. Ismail Z, Peacock SJ, Kovacic L, Hoch JS. Cost-effectiveness impacts cancer care funding decisions in British Columbia, Canada, evidence from 1998 to. Int J Technol Assess Health Care. 2008;5:1-6, 2017.

14. Hoch JS, Dewa CS. Advantages of the net benefit regression framework for economic evaluations of interventions in the workplace: a case study of the cost-effectiveness of a collaborative mental health care program for people receiving short-term disability benefits for psychiatric disorders. J Occup Environ Med. 2014;56(4):441-5.

15. Hoch JS, Dewa CS. Lessons from trial-based cost-effectiveness analyses of mental health interventions: why uncertainty about the outcome, estimate and willingness to pay matters. Pharmacoeconomics. 2007;25(10):807-16.

16. Hoch JS. Net benefit regression. In M. Kattan (editor), encyclopedia of. Med Decis Mak. 2009;2:805-11.

17. Hoch JS, Briggs AH, Willan AR. Something old, something new, something borrowed, something blue: a framework for the marriage of health econometrics and cost-effectiveness analysis. Health Econ. 2002;11(5):415-30

18. O'Brien BJ, Drummond MF, Labelle RJ, Willan A. In search of power and significance: issues in the design and analysis of stochastic costeffectiveness studies in health care. Med Care. 1994;32:150-63.

19. Nixon RM, Wonderling D, Grieve RD. Non-parametric methods for costeffectiveness analysis: the central limit theorem and the bootstrap compared. Health Econ. 2010;19(3):316-33.

20. Hoch JS, Rockx MA, Krahn AD. Using the net benefit regression framework to construct cost-effectiveness acceptability curves: an example using data from a trial of external loop recorders versus Holter monitoring for ambulatory monitoring of "community acquired" syncope. BMC Health Serv Res. 2006;6:68

21. Stinnett AA, Mullahy J. Net health benefits: a new framework for the analysis of uncertainty in cost-effectiveness analysis. Med Decis Mak. 1998 Apr-Jun;18(2 Suppl):S68-80.

22. Bang $H$, Tsiatis AA. Estimating medical costs with censored data. Biometrika. 2000;87:329-43.

23. Chen S, Rolfes J, Zhao H. Estimation of mean health care costs and incremental cost-effectiveness ratios with possibly censored data. Stata J. 2015;15(3):698-711.

24. Hoch JS, Chaussé P. Econometric considerations when using the net benefit regression framework to conduct cost-effectiveness analysis. B. Baltagi and F. Moscone (editors), health econometrics in contributions to economic analysis: Emerald Publishing; 2018.

\section{Publisher's Note}

Springer Nature remains neutral with regard to jurisdictional claims in published maps and institutional affiliations.

Ready to submit your research? Choose BMC and benefit from:

- fast, convenient online submission

- thorough peer review by experienced researchers in your field

- rapid publication on acceptance

- support for research data, including large and complex data types

- gold Open Access which fosters wider collaboration and increased citations

- maximum visibility for your research: over $100 \mathrm{M}$ website views per year

At BMC, research is always in progress.

Learn more biomedcentral.com/submission 